\title{
Acquérir une compétence lectoriale en français langue étrangère et seconde
}

\author{
Estelle Riquois \\ Université de Rouen, LiDiFra EA 4305 \\ estelle.riquois@univ-rouen.fr
}

\section{Introduction}

Dans une classe de français langue étrangère, comme dans une classe de français langue seconde ${ }^{1}$, l'apprenant doit faire appel à différentes compétences pour répondre aux sollicitations de l'enseignant, réaliser les différentes activités d'apprentissage qui lui sont proposées et pouvoir progresser. Dans le champ du FLE/S, ces compétences ont été théorisées par l'approche communicative dans les années 1980 , et il est communément admis aujourd'hui qu'elles sont au nombre de quatre : les compréhensions orale et écrite, les productions orale et écrite.

Les travaux récents du Conseil de l'Europe ont permis d'affiner ces compétences en proposant dans le Cadre Européen Commun de Référence pour les langues (Conseil de l'Europe, 2001) de les considérer désormais comme des «activités langagières ». Ces activités sont les suivantes : production orale ou écrite, réception orale ou écrite, interaction orale ou écrite, et médiation orale ou écrite. Ce nouveau mode de désignation permet de disposer d'outils plus précis destinés à mieux développer les compétences de l'apprenant et à organiser les tâches d'apprentissage de façon plus efficace. Celles-ci sont effectivement identifiées différemment, autorisant l'enseignant à opérer des distinctions plus fines lorsqu'il choisit les activités, comme on peut le constater dans des manuels très récents ${ }^{2}$ mettant en œuvre ces propositions.

De façon complémentaire, la compétence de communication a également été reformulée. Elle regroupe à présent les « compétences communicatives langagières » qui se déclinent sous trois aspects principaux : les compétences linguistiques, sociolinguistique et pragmatique qui peuvent elles aussi se décomposer en différentes composantes et compétences ${ }^{3}$. Or, si les compétences linguistiques ${ }^{4}$ sont bien connues et enseignées dans les classes, il n'en est pas de même pour les compétences sociolinguistique ${ }^{5}$ et pragmatique $^{6}$. Peu d'outils descriptifs sont disponibles à ce jour et ces compétences comportent des subdivisions parfois difficiles à définir. Les manuels de civilisation ne sont pas davantage une aide probante pour l'enseignant, car ils ne sont pas adaptés à l'apprentissage de ces compétences. Ils portent surtout sur l'acquisition de savoirs, alors que les objectifs visés aujourd'hui s'orientent vers l'acquisition complémentaire de savoirs, de savoir-faire, de savoir-être et de savoir-apprendre. Ajoutons enfin que le Cadre européen commun de référence pour les langues (2001) concerne l'ensemble des langues européennes. Pour répondre à cet objectof, il se doit de rester général, ce qui interdit toute description trop précise de ces compétences.

Les auteurs du Cadre européen proposent d'autres redéfinitions, mais les propositions que nous venons d'évoquer invitent déjà à la réflexion. Il semble en effet indispensable de s'interroger plus attentivement sur ces différents aspects des compétences communicatives langagières, et plus largement sur les compétences transversales qui peuvent permettre à l'apprenant d'acquérir une plus grande autonomie dans son usage du français. L'objectif est d'abord descriptif pour déterminer quelles sont ces compétences et quelles sont leurs caractéristiques. Il est ensuite pragmatique, car il s'agit aussi de proposer des outils d'enseignement / apprentissage à destination des enseignants et des apprenants.

\section{Lire : une compétence indispensable en classe de FLE/S}

Pour mener cette réflexion, nous avons choisi de nous intéresser plus particulièrement à une activité langagière qui était déjà une des quatre compétences prises en compte dans le cadre de l'approche 
communicative et qui est omniprésente dans les classes de langue vivante ayant adopté l'approche communicative ou la jeune perspective actionnelle : la réception écrite.

\subsection{Lecture et classe de FLE}

Lire et comprendre l'écrit sont des compétences qui ont une grande importance dans les sociétés actuelles et chaque individu est confronté quotidiennement à des messages tant oraux qu'écrit. L'exclusion d'un de ces domaines d'apprentissage semble alors dommageable pour l'apprenant qui ne peut maîtriser qu'une partie des possibilités d'usage de la langue qui lui sont offertes. L'enseignant peut également pâtir de l'exclusion de l'écrit car il peut s'appuyer sur un manuel pour proposer des activités spécifiques, ou suivre la progression indiquée dans l'ouvrage. Certes, comme le dit Vargas, «à des degrés divers, pour des causes diverses, le manuel est un mauvais outil irrémédiable» $(2006: 27)$. Il reste cependant bien utile pour de nombreux enseignants, qui peuvent aussi avoir recours à des documents authentiques écrits ou à des supports pédagogiques de même nature, ce qui rend indispensable la maîtrise de cette activité langagière qu'est la réception écrite.

Dans de nombreuses classes de langue, cette activité est donc sollicitée en permanence. La lecture de consignes dans le manuel ou dans le livre d'exercice, la lecture d'un document authentique, du titre d'une publicité, des sous-titres si l'enseignant utilise un support vidéo sous-titré, la lecture d'un document sur Internet, projeté sur un écran unique dans la classe ou lu sur un écran individuel, la relecture des réponses fournies pour un exercice ou bien encore la relecture d'un devoir d'expression écrite, toutes ces activités dont la liste n'est pas close - demandent à l'apprenant une maîtrise de la compréhension écrite qui conditionne leur réussite.

Cette activité langagière n'a pourtant pas toujours fait l'objet de discours spécifiques dans les méthodologies qui se sont succédées pendant le $20^{\mathrm{e}}$ siècle. Avant cette période, le texte littéraire et son étude étaient privilégiés pour l'apprentissage du français. Par réaction contre cette méthode dite «traditionnelle» ou «grammaire-traduction», et pour répondre à de nouveaux besoins chez les apprenants (volonté de communiquer oralement, nouveaux modes de déplacement favorisant les voyages...), les didacticiens se sont tournés vers l'oral et son apprentissage. A partir de la méthode directe utilisée dans les classes au début du $20^{\mathrm{e}}$ siècle et, plus tard, dans des méthodologies telles que la méthode audio-orale, ou les méthodologies audiovisuelles, l'oral est devenue la compétence privilégiée des premiers temps de l'apprentissage. Le matériel pédagogique et les activités mises en œuvre par les enseignants portaient donc surtout sur l'acquisition de compétences de réception et de production orale durant les heures d'apprentissage correspondant au niveau 1.

Il apparaît pourtant difficile de se passer totalement de l'écrit lorsqu'on observe les manuels qui s'orientent principalement vers l'acquisition de ces compétences. Ainsi, dans l'avant-propos du manuel Studio $60^{7}$, l'objectif affiché est la maîtrise «des capacités [qui] permett[ent] à l'apprenant de communiquer de façon simple dans des situations de la vie quotidienne » (2001:3). Une grande partie des exercices et des activités est tournée vers l'oral, mais le manuel comporte des consignes rédigées en français dès la première leçon et des exercices d'écoute qui s'appuient sur un support écrit. La dissociation de l'oral et de l'écrit semble alors difficile, comme elle l'est d'ailleurs dans les usages. Un locuteur manie sans cesse l'oral et l'écrit, et la maîtrise unique de l'un de ces deux domaines peut être considérée comme un handicappar de nombreuses sociétés.

L'approche communicative a rééquilibré progressivement l'apprentissage des différentes compétences tout en maintenant la priorité donnée à l'oral dans les premiers temps de l'apprentissage. L'écrit a repris sa place, de même que le texte littéraire qui est aujourd'hui considéré comme un document authentique parmi d'autres. Les supports écrits sont ainsi plus nombreux et peuvent répondre à diverses situations d'enseignement / apprentissage.

Par ailleurs, dans cette méthodologie, la réflexion didactique sur l'écrit et ses approches a bénéficié des travaux menés en psycho cognition et des études faites sur la réception de l'écrit. Les processus de lecture sont désormais mieux connus, et plusieurs didacticiens ont proposés des méthodes d'approche des textes 
destinées à donner des outils de compréhension aux apprenants. Il faut citer, par exemple, les travaux de Moirand (1979) qui propose une « approche globale des textes écrits » intervenant « dès que les étudiants connaissent la transcription de l'oral (et même si leur déchiffrage est encore approximatif au plan phonétique) » (1979: 23). L'approche globale est toujours utilisée aujourd'hui, notamment parce qu'elle est proche d'une lecture authentique. Comme un locuteur natif, l'apprenant découvre le texte progressivement, en exploitant les différents indices qui peuvent faciliter le processus de compréhension. Par des activités d'approche du texte et avant de procéder à sa lecture, l'apprenant produit des hypothèses concernant le thème abordé, le type de texte ou sa structure, ce qui lui permet ensuite de confirmer ou d'infirmer ses hypothèses, et non de découvrir un texte totalement nouveau.

\subsection{Lecture scolaire, lecture loisir, lecture d'apprentissage}

Ces propositions mettent en avant quelques spécificités de la lecture en classe de langues vivantes. On ne lit pas de la même façon lorsqu'on lit un texte dans sa langue maternelle, et lorsqu'on lit un texte en langue étrangère. On ne lit pas non plus de la même façon dans une classe et en dehors de celle-ci. Les stratégies mises en œuvre par l'apprenant sont spécifiques à l'apprentissage d'une langue étrangère ou seconde, et au fait de lire dans une langue qui n'est pas encore tout à fait la sienne.

Dans la classe, le texte lu est aussi support d'apprentissage, ce qui lui confère des caractéristiques et un statut particuliers. Les objectifs de la lecture sont adaptés à la tâche d'apprentissage qui s'appuie sur le texte, et le texte lui-même doit permettre de répondre à ces objectifs. Il est donc choisi pour cela et non en fonction d'un besoin de loisir ou d'information, plus habituel en situation de lecture authentique.

Dans le cadre institutionnel d'une situation d'apprentissage, «savoir lire » se double alors d'un « savoir lire en langue étrangère » qui nécessite des compétences quelque peu différentes. Certains apprenants, peu nombreux, transposent automatiquement en langue étrangère ou seconde les stratégies de lecture qu'ils utilisent en langue première. Leur petit nombre impose néanmoins de déterminer quelles sont les stratégies les plus efficaces pour cette activité, afin de les enseigner aux apprenants qui ne les exploitent pas spontanément. En outre, un strict transfert de compétence ne semble pas se justifier, car les processus de compréhension, d'interprétation des textes et les objectifs de lecture sont différents. Il faut à l'inverse envisager, dans le cadre de la classe de langue vivante, le développement de stratégies spécifiques qui permettent d'accéder à un bon niveau de compréhension et de répondre aux objectifs d'apprentissage.

Mais alors qu'est-ce que lire? Qu'entend-t-on exactement par ce que nous nommons «lecture»? Le décodage d'un texte n'est pas suffisant pour accéder à sa signification. Déchiffrer l'écrit se double d'un processus de compréhension, voire d'interprétation qui ne semble pas dissociable de l'opération de décodage. Il faut par conséquent tenir compte de ces deux aspects et les considérer comme deux faces d'un même objet. Le décodage se fait pour comprendre le texte, et la compréhension passe par ce décodage premier.

Nous suivrons donc le parti pris de Dabène (2001) qui choisit de considérer l'objet « lecture » comme une abstraction qui ne permet pas de rendre compte de ce qui se produit réellement entre le lecteur et le texte. Plusieurs facteurs sont à considérer pour définir ce que peut être la lecture, lecture qui ne peut alors se résoudre à cette appellation unique. Ainsi,

\footnotetext{
il s'agit de prendre en compte les composantes hétérogènes de ce que nous appelons les «situations de lecture » en considérant la lecture comme une activité langagière s'exerçant sur les manifestations scripturales de la langue et produisant du sens pour celui qui est engagé dans cette activité complexe. Elle suppose évidemment une activité de reconnaissance plus ou moins automatisée des graphies et de leur signification (l'activité de décodage ou de déchiffrage), nécessaire mais non suffisante. $(2001: 11)$
}

La distinction entre « savoir lire » et « savoir lire en langue étrangère » correspond alors à deux situations de lecture différentes, qui présentent chacune des particularités et des objectifs singuliers. Elles ne sont pas uniques et on peut opérer bien d'autres distinctions, correspondant chacune à des situations de lecture 
différentes. En fonction de conditions extérieures, d'objectifs variés, de types de texte, la lecture peut être caractérisée et nommée "lecture scolaire», «lecture d'apprentissage», «lecture plaisir», «lecture loisir » ou bien encore « lecture professionnelle ».

Nous conserverons ici les dénominations «lecture scolaire», «lecture d'apprentissage » et «lecture loisir ». La première s'oppose à la troisième dans la mesure où la « lecture loisir » relève du domaine privé, du choix du lecteur, et se pratique en dehors du cadre institutionnel d'apprentissage. La «lecture scolaire », au contraire, peut être parfois une «lecture plaisir», mais le choix fait par le lecteur est en relation avec des propositions du professeur ou est imposé par celui-ci. Quand à la «lecture d'apprentissage », nous la définirons comme une lecture qui répond à un objectif extérieur au texte, dépendant de la tâche d'apprentissage et conçu en relation avec celle-ci et non directement en relation avec le texte. Dans la lecture d'apprentissage, le texte est un support d'apprentissage mis au service de l'acquisition d'une compétence de communication. Les stratégies employées par le lecteur ne peuvent évidemment pas être les mêmes dans ces trois types de lecture.

Cette répartition impose également de distinguer différents types de lecteur. À la «lecture d'apprentissage » correspondent un « lecteur apprenant » et une posture de lecteur spécifique. Il ne s'agit pas, dans un premier temps, d'interpréter le texte ou de le lire en envisageant sa visée esthétique - quand il s'agit d'un texte littéraire - mais de répondre à une consigne qui peut parfois porter uniquement sur l'aspect linguistique du texte.

La «lecture loisir», à l'inverse, attend du lecteur qu'il soit impliqué dans sa relation au texte. Plus souvent associée au texte littéraire, elle engage celui qui lit et attend de lui qu'il coopère comme le définit Eco (1985) lorsqu'il introduit les notions de « coopération interprétative » et d' « interprétation critique ». Il est également possible de citer Iser (1976) pour qui « le texte n'existe que par l'acte de constitution d'une conscience qui le reçoit » $(1976: 49)$. Dans cette perspective, le sujet lecteur ${ }^{8}$ prend toute son importance. Il fait vivre le texte en se plaçant dans une relation personnelle et intime avec ce qui y est exprimé. La littérature n'est cependant pas la seule à attendre du lecteur qu'il se place en tant que sujet réel et agissant de sa lecture. Un texte de presse, un essai polémique ou une publicité sont autant d'écrits qui attendent aussi du lecteur qu'il s'implique dans sa lecture.

La « lecture scolaire », enfin, est plus contrainte, tout en se plaçant à l'intersection des deux précédentes. Elle relève de la lecture d'apprentissage dans la mesure où le choix de la lecture n'est pas laissé à l'élève. Celui-ci doit lire un texte ou un ouvrage imposé pour répondre à une consigne formulée par l'enseignant. Elle relève également de la lecture loisir car elle nécessite une implication identique du lecteur. Il doit se positionner en tant que sujet lecteur et se placer dans un processus de compréhension et d'interprétation de même nature, bien qu'il soit ici guidé par l'enseignant ou par les activités proposées pour accompagner la lecture.

Il est alors possible de représenter ces trois situations de lecture sur la figure suivante, qui fait également apparaitre l'implication attendue du lecteur :

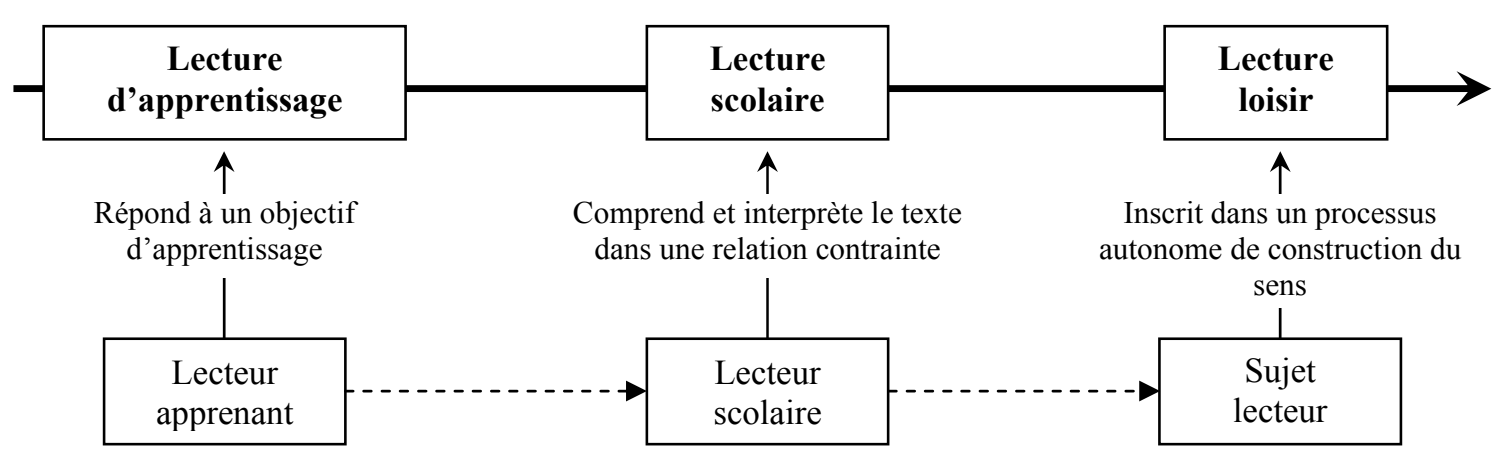

Figure 1. Postures de lecteur en fonction de la situation de lecture considérée. 
Ce schéma général peut être modulé en fonction de la situation d'apprentissage prise en compte. Si l'on considère la problématique spécifique de la lecture en langue étrangère et seconde, qui nous intéresse plus particulièrement ici, les différentes postures de lecteur peuvent être adoptées successivement selon les situations de lecture auxquelles l'apprenant est confronté. Un apprenant de FLE/S peut ainsi être sujet lecteur lorsqu'il est face à un texte écrit dans sa langue maternelle, et lecteur apprenant quand il lit un texte en langue étrangère. Pendant l'apprentissage de cette langue, il s'agit pour lui de progresser de façon à parvenir également à la position de sujet lecteur face à un texte en FLE/S.

À contrario, un apprenant de niveau $\mathrm{C} 1$ ou $\mathrm{C} 2$ peut adopter successivement ou simultanément ces différentes postures. En fonction des tâches d'apprentissage élaborées par l'enseignant et du type de texte proposé, l'apprenant qui dispose de compétences suffisantes peut s'adapter à ce qui lui est demandé et aborder le document écrit en tant que lecteur apprenant, lecteur scolaire ou sujet lecteur. L'exploitation pédagogique d'un texte littéraire, par exemple, appelle un lecteur scolaire dans la mesure où elle répond à une obligation institutionnelle. L'enseignant attend de l'apprenant que celui-ci lise le texte puis réponde à des questions, et l'apprenant agit en fonction de cette situation. Parmi les questions posées, certaines peuvent nécessiter les compétences basiques du lecteur apprenant, comme des demandes portant sur la grammaire ou le vocabulaire, d'autres suscitent une réflexion qui amène l'apprenant à s'exprimer individuellement sur le texte, à donner son opinion ou son interprétation en tant que sujet lecteur. Bien entendu, ces différents temps de l'apprentissage sont successifs, mais pendant la première ou la deuxième lecture, ces postures peuvent se méler et l'observation attentive du texte n'interdit pas son interprétation.

La tripartition considérée ici permet par conséquent de désigner différentes postures, mais également différents types de tâches d'apprentissage. Si la lecture d'apprentissage n'est pas un objectif en soi, la lecture loisir représente en revanche un mode de découverte des textes qui offre à l'apprenant la possibilité d'adopter réellement une posture de lecteur et de disposer des moyens de devenir cet acteur social visé par le Cadre européen commun de référence pour les langues (2001).

Comme nous l'avons évoqué plus haut, l'accès à l'écrit, le décodage des textes n'est pas suffisant pour parvenir à les comprendre. Il en est de même pour la compréhension : elle n'est pas suffisante pour devenir un sujet lecteur. Elle doit se compléter d'une prise de position du lecteur, d'une interprétation et d'une implication dans la lecture qui lient le lecteur et le texte. Ayant fait sien le contenu du texte, le sujet lecteur pourra produire un discours sur celui-ci et se nourrir de ces informations.

\section{Devenir un sujet lecteur}

Il est alors nécessaire de s'interroger sur ce qui caractérise effectivement ce sujet lecteur. Adopter cette posture nécessite des compétences spécifiques et générales mises en œuvre par le sujet lecteur, ainsi qu'une situation qu'il juge propice pour ce faire. Certains textes ne demandent pas d'implication particulière, d'autres sont créés pour cela.

\subsection{Des compétences pour une posture de lecteur}

Dans son ouvrage Le point sur la lecture, Cornaire (1999) expose trois modèles généralement adoptés pour décrire les processus de lecture et de compréhension. Nous retiendrons le modèle dit «interactif» qui se positionne à l'intersection des modèles dits « du bas vers le haut » et " du haut vers le bas (Cornaire, 1999: 22-23). Selon cet auteur, la compréhension est un processus qui fait intervenir conjointement le décodage du texte pendant lequel le lecteur élabore une signification syllabe après syllabe (niveau inférieur vers supérieur), et la formulation d'hypothèses qui sont confirmées, complétées ou modifiées pendant la lecture (niveau supérieur vers inférieur). Pour un lecteur moyen ou expert, différentes sources d'information sont ainsi convoquées et associées afin de parvenir dans un premier temps à la compréhension du texte et dans un second temps à son interprétation. 
Golder et Gaonac'h (2004) vont plus loin et tentent de déterminer ce qui peut faciliter la compréhension pendant la lecture. Si les connaissances du lecteur participent au bon déroulement de cette activité, elles sont accompagnées par d'autres éléments dont le lecteur expert fait un usage constant.

Notre public n'appartient pas à cette catégorie de lecteur, mais la connaissance des stratégies employées à ce niveau de compétence permet de guider les apprenants dans leur apprentissage. De même, l'observation des stratégies courantes chez les lecteurs présentant des difficultés de compréhension met à la disposition du chercheur un panel d'outils potentiellement adaptés aux différents apprenants présents dans un groupe-classe.

Golder et Gaonac'h (2004) ont donc observé chez les lecteurs déficients un recours fréquent au code phonologique car il leur permet de reconnaître les mots lus. La prise d'information graphémique normalement opérée pendant la lecture est déficiente chez ce type de lecteur, ce qui ne leur donne pas les moyens de déchiffrer le texte de manière efficace. Pour y suppléer, il faut avoir recours à ce que les auteurs nomment une « roue de secours » (2004: 43) et utiliser le contexte pour déterminer la nature du mot lu. Cette stratégie n'est pas optimale, car elle ralentit la lecture. Deux opérations doivent se dérouler simultanément, ce qui est coûteux au niveau cognitif et place cette stratégie en position d'aide ponctuelle.

Chez les lecteurs experts, au contraire, déchiffrer le texte et lui associer une signification se fait en quelques millisecondes, car le recours au code phonologique est plus efficace. Le lecteur traduit mentalement le mot en son et s'appuie sur cette représentation pour construire un sens. Le code phonologique semble d'ailleurs être indispensable à toute lecture, bien que l'ensemble des lecteurs ne l'utilise pas avec la même efficacité :

il n'existe pas de lecture purement visuelle, et le codage phonologique (qu'il ne faut pas confondre avec les codages articulatoires qui sont beaucoup plus lents et mettent en jeu le larynx et la bouche) est nécessaire même en lecture silencieuse, ceci que le lecteur soit lent ou rapide. (Golder, Gaonac'h, 2004 : 48)

Ce traitement phonologique contredit toutefois le décryptage lettre par lettre qu'utilisent de nombreux apprenants de FLE /S. Pendant la lecture du texte, les éléments de parole auxquels correspondent les lettres sont isolés mentalement, une image mentale du texte se forme qui permet au lecteur de l' «entendre ». La mémorisation et la compréhension se font ensuite à partir de cette image mentale sonore, filtre décrypté du texte premier.

Cette stratégie de compréhension est considérée par de nombreux chercheurs comme indissociable de la lecture. Toujours utilisée, la médiation phonologique a plusieurs avantages, tels ceux-ci : «allongement du stockage provisoire des informations utiles ; repérage de la structure prosodique ; meilleure résistance aux interférences extérieures »(Golder, Gaonac'h, 2004: 59). C'est pourquoi les lecteurs experts l'utilisent massivement.

Quant aux jeunes lecteurs, ils peuvent rencontrer des difficultés pendant l'apprentissage de la lecture en raison de la complexité de la structure phonologique de la langue. Processus cognitif, la médiation phonologique demande un degré d'abstraction qui peut parfois faire défaut et empêcher la formation d'une représentation mentale idoine. Une des stratégies de compréhension étant défaillante, le lecteur doit s'appuyer sur ce qu'il peut maîtriser, comme le recours au contexte.

Le code phonologique n'est toutefois pas le seul à intervenir dans le processus de compréhension d'un texte écrit en langue étrangère. Selon Gaonac'h (2000), le lecteur apprenant peut avoir recours principalement à trois codes. Le premier, le code logographique, permet à un apprenti lecteur de reconnaitre des mots à partir d'indices plus graphiques qu'orthographiques. Cette perception globale constitue une phase de l'apprentissage de la lecture chez les jeunes enfants qui peut être utilisée par les apprenants dont la langue maternelle utilise un autre système d'écriture. Le code phonologique que nous venons d'évoquer est le deuxième code, tandis que le code orthographique, troisième cité par l'auteur, participe à «la reconnaissance des mots à travers la prise en compte des suites de lettres qui les composent » (2000: en ligne). L'accès au lexique permet également de sélectionner les significations 
adéquates quand un mot peut susciter un doute, et les connaissances générales du lecteur sont convoquées, comme dans une situation de lecture en langue maternelle.

Cette différence de hiérarchie présentée par Gaonac'h (2000) est liée au fait que l'article cité porte spécifiquement sur la lecture en langue étrangère. L'ouvrage de Golder et Gaonac'h (2004) aborde la lecture du point de vue de la langue première. On peut en conclure que le code phonologique, qui revêt une importance particulière pendant la lecture en langue première, passe au second plan lorsque l'apprenant lit en langue étrangère. On en déduit aussi que, comme il a été dit précédemment, les stratégies habituelles en langue première ne sont pas utilisées spontanément en langue étrangère. La compétence de décodage est la seule qui soit disponible immédiatement pour les apprenants de français langue étrangère qui ont appris à lire dans leur langue première.

Il serait simple de voir l'apprenant qui lit un texte en langue étrangère comme un lecteur déficient qui n'utiliserait que les stratégies propres à ce type de lecteur. Bon ou moyen lecteur dans sa langue première, il deviendrait déficient en langue étrangère. On voit immédiatement que cela ne peut être aussi radicale. Cornaire (1999) indique que le lecteur apprenant de langue étrangère fait de fréquents retours en arrière pour valider ou ajuster sa compréhension, ce qui est particulièrement coûteux en temps. Il fait aussi appel à la subvocalisation et déchiffre le texte de façon linéaire, lettre par lettre, avant d'isoler des séquences, ce qui surcharge la mémoire à court terme et interdit toute tâche plus complexe.

Ces stratégies peu rentables s'expliquent par la limitation des connaissances linguistiques de l'apprenant. Sa connaissance partielle de la grammaire interdit les anticipations, tout comme la restriction de son lexique. En langue seconde, l'apprenant semble également avoir recours plus longtemps à la subvocalisation.

Enfin, il faut évoquer un dernier élément proposé par Cornaire (1999) pour expliquer les difficultés des apprenants dans la classe de langue étrangère : l'inquiétude. Peu théorisée à ce jour, l'affectivité est pourtant un facteur déterminant de l'apprentissage. Placé au sein d'un groupe plus ou moins familier, engagé dans une relation avec la classe et l'enseignant, l'apprenant peut se sentir en situation d'infériorité par rapport à sa maîtrise estimée de la langue cible. Il doit alors trouver sa place dans le groupe et face à l'enseignant, et gérer son image. En confiance, la prise de risque et de décision est facilitée, tandis qu'à l'inverse, pour un apprenant peu sûr de lui, l' « inquiétude, [la] nervosité se manifestent au contact des textes étrangers et la tâche devient un obstacle presque infranchissable » $(1999: 51)$.

Il est donc envisageable que le manque de confiance en soi dû à la langue étrangère, la situation de lecture, le climat existant dans la salle de classe ait un rôle dans la compréhension du texte. Les compétences de l'apprenant sont déterminantes, mais elles ne sont pas seules à intervenir dans ce processus. Il appartient à l'enseignant d'instaurer un climat de confiance dans sa classe, puis de sélectionner les textes exploités en fonction des compétences observées chez les apprenants et des stratégies qu'ils mettent en place. Les activités proposées vont ensuite permettre de développer d'autres stratégies, ou vont laisser du temps à l'apprenant pour lire le texte sans entrave et prendre de l'assurance. L'échange d'expérience entre apprenants et la verbalisation des stratégies de chacun peuvent aussi être des pistes de réflexion pour le didacticien, puis pour l'enseignant.

\subsection{Le sujet lecteur de littérature}

\subsubsection{Une pluralité de lectures}

Nous avons évoqué la lecture, jusqu'à présent, comme une activité se rapportant à un type générique de document : les textes écrits. Cette catégorie n'est cependant pas homogène, comme on peut l'observer quand on étudie l'ensemble des documents authentiques écrits. De l'article de presse au texte littéraire, en passant par la publicité, le lecteur dispose d'un choix très vaste, tant par la taille du texte que par le type de support. 
Afin de faire des analyses et des propositions plus précises, il peut être utile de s'intéresser à un type de lecture et un type de texte en particulier. À partir de cette observation restreinte, des transpositions de compétences et de stratégies sont ensuite envisageables vers d'autres types de textes.

L'objectif qui guide notre choix vise à sélectionner un type de lecture identifiable, et nécessitant des compétences spécifiques. Il s'agit également de choisir un type de texte ciblé, c'est pourquoi nous nous sommes orientés vers l'analyse de la lecture littéraire. Le processus de communication qu'implique la lecture d'un texte littéraire est singulier, tout en permettant à l'apprenant d'utiliser des stratégies transposables à d'autres types de texte. Cette lecture invite également le lecteur à adopter plus facilement une posture de sujet lecteur, dénomination d'ailleurs surtout utilisée au sujet de la lecture littéraire.

Par ailleurs, dans la classe de FLE/S, le texte littéraire est un type de document particulier. Classé parmi les documents authentiques, il n'a pas le même statut pour l'apprenant qu'un texte de presse ou qu'une recette de cuisine. L'enseignant lui-même peut parfois choisir de ne pas l'exploiter en raison de son caractère patrimonial, du rejet qu'il peut susciter dans la classe, ou d'une difficulté supposée (ou réelle selon les textes) de la langue littéraire. La littérature véhicule un système de valeur et prend place dans un système culturel qui peut avoir une incidence sur la réception du texte et sur l'inquiétude que nous évoquions précédemment.

Pourtant, son exploitation pédagogique peut permettre la mise en place de tâches d'apprentissage correspondant aux nouvelles orientations proposées par la perspective actionnelle. Réinvestie par des exploitations pédagogiques innovantes, prenant en compte les propositions conjointes du FLM et du FLE, la lecture littéraire peut être une réelle source d'apprentissage dans de nombreuses situations d'enseignement / apprentissage.

\title{
3.2.2 Lecture littéraire et apprentissage
}

La plupart des théoriciens de la lecture littéraire appartiennent au domaine du français langue maternelle. Les propositions pédagogiques qui en découlent sont par conséquent adaptées à des situations d'enseignement / apprentissage qui doivent être repensées pour trouver leur place dans les classes de FLE/S. Les croisements entre ces deux champs de recherche sont malgré tout féconds et la réflexion ne peut que gagner à trouver un terrain commun.

Ainsi, nous retiendrons ici les propositions de Dufays, Gemenne, Ledur (2005) et de Rouxel (1996) qui proposent d'envisager la lecture littéraire du point de vue du lecteur, entendue comme un sujet agissant. Pour Dufays (1994), le caractère littéraire d'un texte est un effet du lecteur :

\begin{abstract}
le littéraire réside moins dans le texte que dans sa lecture. [...] l'idée que la littérature est une composante immanente à un certain type de textes conduit à une impasse ; il faut affirmer avec force que la littérature est un effet de lecture qui dépend de l'attitude qu'on adopte à l'égard du texte. Aucun texte n'est littéraire en soi. (1994:213, l'auteur souligne)
\end{abstract}

Cette position extrême montre l'importance du regard du lecteur sur le texte et de la posture qu'il adopte. L'actualisation du texte devient double, le lecteur le fait exister par sa lecture, et lui permet d'accéder à un statut de texte littéraire par le regard qu'il lui porte.

Dans le cadre de la classe, ce postulat revêt une importance particulière car le paratexte du manuel quand le texte y figure - ou les explications de l'enseignant vont conditionner l'adoption de cette posture. Comme le montre Poslaniec (2002), le caractère littéraire d'un texte seul et isolé peut être difficile à identifier. Le paratexte est donc essentiel à ce processus qui précède la lecture. Guidé vers l'identification $\mathrm{du}$ texte, le lecteur apprenant peut adopter les stratégies adéquates et aller au-delà de la simple compréhension.

La dénomination «lecture littéraire» recouvre par ailleurs différentes acceptions. Elle peut désigner uniquement la lecture-compréhension du support « texte littéraire », ce qui nous semble insuffisant. Elle 
peut aussi être conçue comme un va-et-vient dialectique entre deux modes de lectures : la distanciation et la participation (Dufays, Gemenne, Ledur, 2005).

La distanciation est ce que Canvat (2007) nomme « la régie de compréhension» (2007: 23). Le lecteur suspend la valeur référentielle du texte, il recherche les symboles auxquels celui-ci a recours, il l'observe comme ayant une polysémie maximale. La littérature est considérée comme ce qu'elle est : de la fiction et une illusion de réalité construite par un auteur en utilisant des procédés techniques spécifiques. Lire de cette façon offre des avantages au niveau pédagogique, cela « va de pair avec l'accès à la symbolisation, la mobilisation d'activités cognitives et culturelles variées, la construction d'un sens et d'une culture communs » (Dufays, Gemenne, Ledur, 2005 : 92). Cela ne laisse aucune place, cependant, au plaisir de lire. Le processus affectif, constitutif de la lecture authentique, est mis de coté dans cet exercice artificiel de lecture, artificiel parce qu'incomplet.

À l'opposé de cette conception, la lecture conçue comme une participation restitue l'illusion référentielle et l'implication du lecteur. C'est la « régie de progression » de Canvat (2007: 23) ou la lecture ordinaire, celle qui ne se soucie que du plaisir du lecteur. Impliqué dans le texte, il ne pratique aucune analyse critique, ne décode aucun symbole et accepte tout ce qui est écrit au fil du texte. Dans ce mode de lecture, «les valeurs associées [...] sont celles de la lisibilité (l'unité, la cohérence), de la conformité aux codes génériques, du rapport à la réalité, de la conformité éthique et de la référentialité » (Dufays, Gemenne, Ledur, 2005 : 92). Le lecteur peut choisir le livre en fonction du plaisir qu'il en attend et a à sa disposition l'ensemble de la production littéraire, restreinte comme élargie. Ce type de lecture est d'ailleurs celui que l'on associe généralement aux ouvrages appartenant au champ de production élargie, la distanciation étant théoriquement l'apanage du champ de production restreinte. Sans doute est-il possible d'envisager une autre répartition, car un roman sentimental peut aussi être lu en adoptant un mode de lecture distancié. Dans le contexte d'enseignement / apprentissage d'une langue étrangère, ce mode de lecture amène l'apprenant-lecteur à faire appel à ses émotions et à son imagination. Il peut ainsi concevoir la lecture comme une activité qui lui apporte une satisfaction psycho affective. Cependant, dans une classe, cette lecture présente le désavantage d'être justement trop ordinaire. En s'appuyant sur le plaisir et le goût de lire, elle n'apporte aucune spécificité et, donc, aucune compétence nouvelle, si ce n'est l'accès au livre, déjà important.

C'est pour cette raison que la lecture littéraire doit être conçue comme un équilibre harmonieux entre ces deux conceptions, notamment en classe de langue étrangère. La distanciation demande de nombreuses compétences que les apprenants n'ont pas toujours, y compris en langue maternelle, et le plaisir de la lecture propre à la participation peut être un objectif intéressant dans une situation où le développement de l'autonomie est important. Il faut alors adopter un mode de lecture où le lecteur s'implique affectivement dans l'activité qu'il pratique tout en conservant une distance critique, où les apprentissages sont possibles, conjointement à l'appréciation de cette activité. Facteur de motivation, l'aspect plaisant de la lecture des textes littéraires ne peut que faciliter les tâches communicatives et les projets mis en place autour de ce type de document.

\section{De la compétence de lecture à la compétence lectoriale}

À partir de ce processus particulier de lecture littéraire dialectique, il est possible d'envisager la construction d'une compétence dite «lectoriale » qui irait au-delà de ce que l'on nomme communément la compétence de lecture.

\subsection{Définir une compétence lectoriale}

Nous l'avons rapidement abordé ci-dessus, lire un texte littéraire nécessite de s'appuyer sur le paratexte qui l'accompagne. La lecture en classe comme à l'extérieur de celle-ci ne se limite donc pas à l'espace physique du texte, mais va au-delà. Elle s'appuie sur l'objet-livre lorsqu'il existe, sur les indications de la présentation du manuel, sur les informations ajoutées par l'enseignant quand le texte se présente sous forme de photocopie. Elle exploite aussi les informations connues par l'apprenant et plus largement par le 
lecteur. Son savoir encyclopédique est mis à contribution, ses lectures antérieures, ses connaissances dans le domaine littéraire...

La compétence de lecture va alors dépasser le « savoir lire » et s'étendre aux savoirs comme aux savoirfaire. Lire une première de couverture et savoir interpréter les indications fournies par l'éditeur peut s'avérer bien difficile pour un apprenant habitué à un autre système culturel et d'autres codes éditoriaux. Le jaune et le noir sont dédiés en France au roman policier, le gris à la science-fiction et le rose et le rouge aux romans sentimentaux. Mais qu'en est-il au Japon ou en Russie par exemple?

Ces informations sont pourtant primordiales si l'on considère les écrits de Schaeffer (1989) pour qui « le régime lectorial de la généricité, avant de répondre à un souci classificatoire, est présent dans tout acte de réception, en tant que toute réception implique une interprétation et que celle-ci ne saurait se faire en dehors d'un horizon générique » (1989: 151). L'identification générique du texte conditionnant sa lecture, il importe que l'apprenant dispose d'informations lui permettant d'opérer cette identification.

En élargissant encore l'horizon de nos observations, nous voyons que les compétences d'un lecteur expert francophone natif comportent aussi des informations portant sur les lieux associés au livre. L'objet-livre est mis à disposition du lecteur dans des lieux eux-mêmes régis par des codes spécifiques dont la méconnaissance peut freiner l'accés au texte. Qu'il s'agisse d'une librairie, d'une bibliothèque municipale ou de la bibliothèque d'un centre de langue, il peut être intéressant de maîtriser les différents types de classification utilisés, les méthodes d'emprunt, les prix d'achat... informations qui feront de l'apprenant un lecteur autonome tout d'abord, puis un acteur social apte à mener à bien l'achat puis la lecture et l'interprétation d'un texte.

Ces savoirs et savoir-faire, peu enseignés dans les classes, viennent compléter la compétence de lecture. Ils nécessitent alors une appellation différente qui mette en avant le lecteur et les compétences qu'il acquiert par leur maîtrise. C'est le cas de la dénomination «compétence lectoriale » qui reprend la terminologie utilisée par Schaeffer à propos de l'aspect générique de cette compétence. Elle peut être figurée sous la forme de cercles concentriques, car au niveau spatial comme au niveau de la lecture ellemême, elle s'approche du texte ou s'en éloigne, mais en le conservant toujours en son centre.

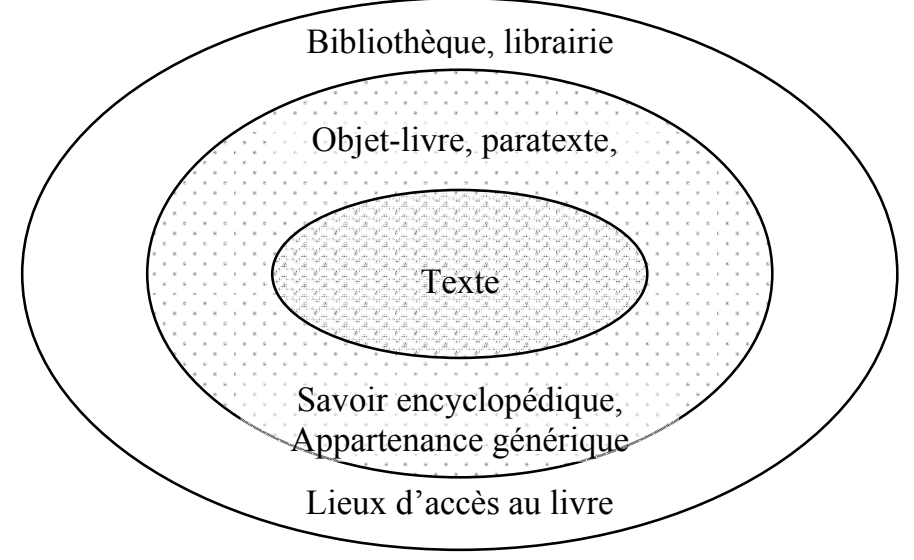

Figure 2. Représentation de la compétence lectoriale.

Le développement de cette compétence lectoriale nécessite de mettre en place des tâches d'apprentissage qui vont bien au-delà de la lecture d'un extrait photocopié. Il est possible d'envisager la création d'une bibliothèque dans la classe, si l'on ne dispose pas d'un lieu de ce type dans l'établissement où ont lieu les cours de FLE/S, ou d'organiser un déplacement dans une bibliothèque, par exemple, lorsque les apprenants sont en situation d'immersion dans un milieu francophone. Il importe en tout cas de créer un espace à la lecture personnelle et individuelle des apprenants. 
La pédagogie de projet introduite récemment par la perspective actionnelle ouvre un espace propice à la mise en place de tâches de ce type. Elle vise un objectif précis, une réalisation concrète, parfois, mettant en œuvre plusieurs compétences différentes et pouvant se dérouler sur de nombreuses séances. Pourquoi ne pas envisager des projets de lecture commune, des lectures individuelles restituées sous forme d'affichage, d'exposés, de débats... qui fassent intervenir tout le groupe mais qui laissent à chacun le choix de la gestion du temps de lecture.

\subsection{Des outils indispensables pour l'autonomie de l'apprenant}

En guise de conclusion, il importe d'insister sur les possibilités d'autonomie offertes à l'apprenant par le biais de l'acquisition de cette compétence lectoriale. Dans de nombreuses situations d'enseignement / apprentissage du FLE/S, le livre est l'une des seules sources d'accès à la langue cible. Peu de locuteurs sont parfois disponibles pour mener des conversations en français, l'accès à l'Internet peut être limité, mais les bibliothèques des universités ou des centres de langues sont à la disposition des apprenants pour parfaire leur maîtrise de la langue française.

Le texte littéraire garde également un prestige qui peut valoriser l'apprenant, tandis que la lecture des romans les plus récents, appartenant à l'actualité littéraire, le rapproche des pratiques françaises. Toutefois, là encore, il lui faut connaître les modalités d'accès à ces publications diverses et variées. Certes, une bibliothèque est un espace où le regard de l'autre se fait moins pesant. L'apprenant a le temps de chercher, de se familiariser avec les livres qu'il a face à lui, mais pour entrer dans ce lieu, il est préférable qu'il dispose de compétences qui le rassureront et lui donneront les moyens de réellement choisir ce qu'il souhaite lire. En se développent, la compétence lectoriale de l'apprenant lui permet donc de disposer d'un accès de plus en plus large à la littérature française, et cette diversité plus grande enrichit de la même façon la compétence lectoriale.

Enfin, il faut signaler l'intérêt de cette compétence pour d'autres types de documents écrits. Nous avons abordé le texte littéraire pour mener cette analyse, mais il est également possible d'envisager de l'appliquer à la lecture de la presse française et francophone, ou à d'autres types de documents écrits. Chaque domaine possède ses caractéristiques et leur connaissance facilite leur lecture. De même, la posture de lecteur n'est pas propre au texte littéraire. De nombreux types d'écrits attendent du lecteur qu'il prenne position et s'implique dans sa lecture.

\section{Références bibliographiques}

Adam, J.-M. \& Heidmann U. (2006). Six propositions pour l'étude de la généricité. Baroni, R. \& Macé M. (dir.). Le Savoir des genres. Rennes : Presses Universitaires de Rennes, 21-34.

Albert, M.-C. \& Souchon M. (2000). Les textes littéraires en classe de langue. Paris : Hachette.

Baroni, R. \& Macé, M. (dir) (2006). Le Savoir des genres. Rennes : Presses Universitaires de Rennes.

Barthes, R. (1973) : Le plaisir du texte. Paris : Seuil.

Bayard, P. (2007). Comment parler des livres que l'on n'a pas lus ? Paris : Minuit.

Bourdet, J.-F. (1988). Texte littéraire : l'histoire d'une désacralisation. Le Français dans le monde, $n^{\circ}$ spécial, 144148.

Canvat, K. \& Legros, G. (2004). Les valeurs dans / de la littérature. Namur : Presses universitaires de Namur.

Canvat, K. (1999). Enseigner la littérature par les genres. Pour une approche théorique et didactique de la notion de genre littéraire. Bruxelles : De Boeck, Duculot.

Canvat, K. (2007). Lire du côté de chez soi. Réhabiliter la lecture "ordinaire". Bemporad, C. \& Jeanneret T. (dir.). Lectures littéraires et appropriation des langues étrangères. Études de Lettres, 4, $19-52$.

Cicurel, F. \& Moirand, S. (1990). Apprendre à comprendre l'écrit. Hypothèses didactiques. Gaonac'h, D. (dir.). Acquisition et utilisation d'une langue étrangère, Approche cognitive. Paris : Hachette, 147-157. 
Cicurel, F. (1991). Lectures interactives en langue étrangère. Paris : Hachette.

Cicurel, F. (2007). Postures et médiations pédagogiques pour la lecture littéraire. Bemporad, C. \& Jeanneret T. (dir.). Lectures littéraires et appropriation des langues étrangères. Études de Lettres, 4, 155-174.

Compagnon, A. (2004). Dévaluations de la littérature. Canvat, K. \& Legros, G. (dir.). Les valeurs dans / de la littérature, Namur : Presses universitaires de Namur, 75-88.

Conseil de l'Europe, (2001). Cadre européen commun de référence pour les langues. Apprendre, enseigner, évaluer. Paris : Didier.

Cornaire, C. (1999). Le point sur la lecture. Paris : Clé international.

Dabène, M. (2001). Situations de lecture et quête du sens: cheminements et fractures. Cahiers du français contemporain, 7, 9-20.

Dufays, J.-L. (1994). Stéréotype et lecture. Liège : Mardaga.

Dufays, J.-L. (2001). Le stéréotype, un concept-clé pour lire, penser et enseigner la littérature. Marges Linguistiques, Actes du colloque d'Albi « Langage et signification », 1-14.

Dufays, J.-L. (2004). La dialectique des valeurs : le jeu très ordinaire de l'évaluation littéraire. Canvat, K. \& Legros G. (dir.). Les valeurs dans / de la littérature. Namur : Presses universitaires de Namur, 103-129.

Dufays, J.-L., Gemenne L., Ledur D. (2005). Pour une lecture littéraire : histoire, théories, pistes pour la classe. Bruxelles : De Boeck.

Eco, U. (1985). Lector in fabula, le rôle du lecteur ou la coopération interprétative dans les textes narratifs. Paris : Le Livre de Poche.

Gaonac'h, D. (2000). La lecture en langue étrangère : un tour d'horizon d'une problématique de psychologie cognitive. Acquisition et interaction en langue étrangère, 13, [en ligne]. http://aile.revues.org/document970.html

Genette, G. (1987). Seuils. Paris : Seuil.

Gervais, B. \& Bouvet, R. (dir) (2007). Théories et pratiques de la lecture littéraire. Québec : Presses de 1'Université du Québec.

Giasson, J. (2008). La compréhension en lecture. Bruxelles : De Boeck.

Golder, C. \& Gaonac'h, D. (2004). Lire \& comprendre. Psychologie de la lecture. Paris : Hachette éducation.

Iser, W. (1976). L'acte de lecture. Théorie de l'effet esthétique. Bruxelles : Mardaga.

Jouve, V. (1993). La lecture. Paris : Hachette supérieur.

Lane, P. (1992). La Périphérie du texte. Paris : Nathan Université.

Moirand, S. (1979). Situations d'écrit, compréhension / production en français langue étrangère. Paris: Clé international.

Picard, M. (1986). La lecture comme jeu. Paris : Minuit.

Poslaniec, C. (2002). Vous avez dit littérature? Paris : Hachette.

Reuter, Y. (1981). L'objet-livre. Pratiques, 32, 104-113.

Reuter, Y. (1985). La quatrième de couverture, problèmes théoriques et pédagogiques. Pratiques, 48, 53-70.

Rouxel, A. \& Langlade, G. (2004). Le sujet lecteur. Lecture subjective et enseignement de la littérature. Actes du colloque "Sujets lecteurs et enseignement de la littérature ». Rennes : Presses universitaires de Rennes.

Rouxel, A. (1996). Enseigner la lecture littéraire. Rennes : Presses Universitaires de Rennes.

Schaeffer, J.-M. (1989). Qu'est-ce qu'un genre littéraire? Paris : Seuil.

Séoud, A. (1997). Pour une didactique de la littérature. Paris : Crédif, Hatier / Didier.

Souchier, E. (1998). L'Image du texte, pour une théorie de l'énonciation éditoriale. Merzeau, L. (dir.). Les Cahiers de médiologie $n^{\circ} 6,137-145$. 
Vargas, C. (2006). Les manuels scolaires. Imperfections nécessaires, imperfections inhérentes et imperfections contingentes. Lebrun, M. (dir.). Le manuel scolaire, un outil à multiples facettes. Québec, Presses universitaires de Québec, 13-35.

Vlad, M. (2006). Lire des textes en français langue étrangère à l'école. Cortil-Wodon : E.M.E.

\footnotetext{
${ }^{1}$ Nous utiliserons désormais l'abbréviation FLE/S pour désigner les champs du français langue étrangère et du français langue seconde, mais une distinction est nécessaire entre ces deux champs car l'accès à l'écrit ne s'y fait pas selon les mêmes modalités. Ils désignent effectivement deux types de public dont les caractéristiques sont différentes et pour lesquels l'acquisition d'une compétence lectoriale diffère sensiblement.

${ }^{2}$ Le manuel Scénario, par exemple, qui applique les modifications proposées par le Cadre européen commun de référence pour les langues, propose différents types d'exercices et d'activités correspondant à cette nouvelle répartition, ce qui enrichit le choix proposé à l'enseignant (Dubois, A.-L. \& Lerolle, M. (2008). Scénario, méthode de français. Paris : Hachette).

${ }^{3}$ Ces compétences communicatives langagières sont définies dans le chapitre $\mathrm{V}$ du Cadre européen commun de référence pour les langues (2001: 86-101).

${ }^{4}$ Ces compétences linguistiques sont détaillées comme suit: compétence lexicale, compétence grammaticale, compétence sémantique, compétence phonologique, compétence orthographique, compétence orthoépique (Conseil de l'Europe, $2001: 86-93$ ).

${ }^{5}$ La compétence sociolinguistique est détaillée comme suit: marqueurs des relations sociales, règles de politesse, expressions de la sagesse populaire, différences de registre, dialecte et accent (Conseil de l'Europe, 2001 : 93-96).

${ }^{6}$ La compétence pragmatique est détaillée comme suit: compétence discursive, compétence fonctionnelle, compétence de conception schématique (Conseil de l'Europe, 2001 : 96-100).

${ }^{7}$ Lavenne, C., Bérard, E., Breton, G., Canier, Y., Tagliante, C. (2001). Studio 60, méthode de français, niveau 1. Paris : Didier.

${ }^{8}$ La notion de « sujet lecteur » est notamment abordée dans l'avant-propos des actes du colloque Sujets lecteurs et enseignement de la littérature (Rouxel, A. \& Langlade, G., 2004).
} 